\title{
Assessment of Specificity of the Badcamp Agility Test for Badminton Players
}

\author{
by \\ Luiz de França Bahia Loureiro Jr1,2,3, Mário Oliveira Costa Dias², \\ Felipe Couto Cremasco' ${ }^{2}$ Maicon Guimarães da Silva², Paulo Barbosa de Freitas ${ }^{3}$
}

The Badcamp agility test was created to evaluate agility of badminton players. The Badcamp is a valid and reliable test, however, a doubt about the need for the use of this test exists as simpler tests could provide similar information about agility in badminton players. Thus, the aim of this study was to examine the specificity of the Badcamp, comparing the performance of badminton players and athletes from other sports in the Badcamp and the shuttle run agility test (SRAT). Sixty-four young male and female athletes aged between 14 and 16 years participated in the study. They were divided into 4 groups of 16 according to their sport practices: badminton, tennis, team sport (basketball and volleyball), and track and field. We compared the groups in both tests, the Badcamp and SRAT. The results revealed that the group of badminton players was faster compared to all other groups in the Badcamp. However, in the SRAT there were no differences among groups composed of athletes from open skill sports (e.g., badminton, tennis, and team sports), and a considerable reduction of the difference between badminton players and track and field athletes. Thus, we concluded that the Badcamp test is a specific agility test for badminton players and should be considered in evaluating athletes of this sport modality.

Key words: badminton, racket, sport, quickness, uncertainty, reaction time.

\section{Introduction}

Team and racket sports require fast movements with continuous changes of direction triggered by stimuli from the opponents, the environment, or both. It makes the physical capability known as agility one of the most important for successful performance in such sport modalities. Previous studies have recommended the use of specific agility tests for various sports like netball (Farrow et al., 2005), Australian football (Sheppard et al., 2006; Veale et al., 2010), tennis (Monte and Monte, 2007), basketball (Mikołajec et al., 2013), as well as football and futsal (Benvenuti et al., 2010). A common feature of these tests is that they challenge participants to perform shifts and changes of direction guided by visual stimuli. However, the demand for expensive electronic equipment or the lack of a specific test validated for a given sport eventually leads coaches to use generic tests of changes of direction such as the shuttle run agility test (SRAT- Adam et al., 1988; Salaj and Markovic, 2011; Sporis, et al., 2010) and zig-zag/slalom type tests (Little and Williams, 2005; Sporis et al., 2010; Vescovi et al., 2008) for agility assessment. Despite these tests being easy to perform and of simple application, the movements performed in these tests do not reflect those performed during most of sports practice

1 - Centro Universitário Católica de Quixadá, Quixadá, CE, Brazil.

2 - Associação Esportiva Viva o Badminton, Campinas, SP, Brazil.

3 - Interdisciplinary Graduate Program in Health Sciences, Cruzeiro do Sul University, São Paulo, SP - Brazil. 
sessions and competitions.

Badminton is a racket sport that requires from players quick movements towards a projectile (i.e., shuttlecock), which can be directed to any place on the court and can displace at a high speed (Hussain, 2011; Tsai and Chang, 1998). As the opponents could hit and send the shuttlecock anywhere in the court, both the players' capacity to change the whole body movement direction (Tiwari et al., 2011) and their reaction times (Cheng et al., 2006; Loureiro Jr. and de Freitas, 2012) are critical for success in this sport. Indeed, a study performed with Indian badminton players revealed that performance of a task consisting of change of direction was more related to the performance of the players on the court $(r=0.83)$ than other physical capacities such as muscle resistance $(\mathrm{r}=0.75)$, muscle strength $(\mathrm{r}=$ $0.69)$, linear running speed $(\mathrm{r}=0.67)$, and power $(\mathrm{r}$ $=0.55$ ) (Tiwari et al., 2011). Thus, evaluation of agility appears to be important for badminton coaches to check their players' athletic status, predict future performance in competitions, and even detect talented players.

Recently, a test to evaluate agility in badminton athletes, which was named Badcamp, was developed and it achieved high rates of construct and concurrent validity as well as testretest reliability (Loureiro Jr. and de Freitas, 2016). The confirmation of concurrent validity was performed against the SRAT, which is a test largely used by coaches and sport scientists interested in assessing agility and, mainly, because the SRAT does not take into account the players' reaction time. Loureiro Jr. and de Freitas (2016) found that performance of badminton players in the Badcamp shared $69 \%$ of the variability with performance in the SRAT. If the SRAT could account for a large amount of the variability of the Badcamp's performance, why should this new test be performed to assess agility in Badminton players? In order to test the specificity of the Badcamp agility test for badminton players we compared the performance of badminton players with athletes from other sports in both the Badcamp and the SRAT. We hypothesized that badminton players would show better performance than athletes from other sports in the Badcamp, yet this performance would not be observed in the SRAT, which would demonstrate the specificity of the Badcamp test for the assessment of agility in badminton players.

\section{Material and Methods}

\section{Participants}

Sixty-four young male and female athletes between 14 and 16 years old participated in this study. They were divided into 4 groups of 16 participants each ( 8 males and 8 females) according to their practiced sport: badminton, field tennis, team sport (volleyball and basketball) and track-and-field (Table 1). All participants had at least 2 years of training, one year of official competitions in their respective sport and no recent history (i.e., 6 months) of musculoskeletal injury. We selected field tennis players because they also use a racket as badminton players. Basketball and volleyball players were selected because as they use their hands to manipulate a projectile (i.e., a ball) during practice and competition. Track and field athletes competing in sprint running and jumping events were selected as they are supposed to have high levels of muscle power and running skills. The study was approved by the Human Research Ethics Committee of the Universidade Cruzeiro do Sul (São Paulo, SP, Brazil) and all participants and their parents or guardians had to sign the informed consent form before starting the tests.

\section{Experimental procedure}

The tests were performed in athletes' practice sites, before their training sessions. It was ensured that the floor on which the tests were conducted were cleaned and dried to prevent accidental slips. Sports shoes commonly used in practice sessions were used to perform the tests. Before testing, all athletes performed typical warm-up activities in order to not hinder their performances. Firstly, participants performed the Badcamp agility test (Loureiro Jr and de Freitas, 2016) and afterwards they performed the SRAT from EUROFIT (Adam et al., 1988). A five-minute rest interval was given to the participants between the performance of the Badcamp and SRAT.

The Badcamp is fully described in Loureiro Jr. and de Freitas (2016). Briefly, this test is performed in a rectangular area of 5.6 by $4.2 \mathrm{~m}$ $(\mathrm{LxW})$. In the center of this area the participant's starting position is demarcated with a rectangle measuring 0.7 by $1.4 \mathrm{~m}(\mathrm{LxW})$. This rectangle is divided into two parts ( 0.7 by $0.7 \mathrm{~m}$ square) in which participants must place each foot. Six 
targets composed of $1.2 \mathrm{~m}$ high inflatable towers are placed on the edges of the test area; four on each corner and two in the middle of each longer side and aligned with the center line of the rectangular area. A luminous panel measuring 0.67 by $0.52 \mathrm{~m}$ is placed in front of the starting position, half meter out of the testing rectangular area (Figure 1A). This panel contains six LED arrows that indicate the direction the participant should run in order to reach the target. The arrows pointing upward correspond to the front targets, the ones pointing downward correspond to the rear targets, and the ones pointing to left and right correspond to the left and right middle targets, respectively. The panel is connected to an integrated circuit that controls the arrows lighting. The arrows light randomly without repetition, one at the time, until all six arrows have been lit. A push button connected to the circuit is placed just in front of the starting position area and is used to control the arrows lighting and to start and end a digital chronometer attached to the panel and used to register the time to perform the test.

The participant was instructed to stay in the starting position area, start the test by pressing the button switch when he/she was ready to do so, and run as fast as possible. The test started when the participant pressed the push button switch for the first time. This first touch lit the first arrow and the participant ran towards the corresponding target, touched it, immediately went back to the starting position area, and pressed the switch again. The second switch pressing lit the second arrow and showed the new target that should be touched. The test ended when the participant finished touching all six targets and pressed the switch for the $7^{\text {th }}$ and last time. Altogether each participant performed 12 movements, six going toward the target and six going back to the starting position, resulting in a running distance of around $36.4 \mathrm{~m}$.

Each participant performed the test three times and the shortest time was considered for further analysis. A 1 min rest interval was given between trials. Before the first valid trial, each participant performed the test at moderate speed to familiarize with the task. If the participant committed errors (e.g., run towards and touch the wrong target), the trial was stopped and repeated. Each participant spent around $8 \mathrm{~min}$ to be assessed using the Badcamp agility test, counting from the beginning of the familiarization trials to the end of the last trial, with the resting time included.

For the SRAT, two $1.2 \mathrm{~m}$ parallel lines, 5 $\mathrm{m}$ distant from each other, were drawn on the floor (Figure 1B). The participant was asked to stand before one of the lines and, after the experimenter signal, run towards the other line, place both feet beyond this line, turn around (180 degrees), return to the starting line, and repeat it four times. Thus, the participant ran back and forth five times each, resulting in a total distance cover of $50 \mathrm{~m}$. The test execution time used as the dependent variable was recorded by the experimenter with a manual chronometer. The shortest time in two trials was used to define participant's performance in the SRAT (Adam et al., 1998). The time needed to perform the whole SRAT (two trial plus resting time) was around 4 min.

\section{Statistical analyses}

After confirmation that the data presented normal distribution (Shapiro-Wilk test) and homogeneity of variance (Levene's test), we performed two one-way (group) analyses of variance (ANOVA), one for the time to perform the Badcamp and one for the time to perform the SRAT. Post-hoc tests with Bonferroni adjustments were carried out to assess the differences between groups. The alpha value was set at 0.05 and the statistical power at 0.8 .

\section{Results}

Figure 2 shows the means and respective standard deviations of the time taken to perform the Badcamp agility test (left panel) and the SRAT (right panel) for all studied groups. ANOVA revealed a main effect of group on the time to perform the Badcamp $[\mathrm{F}(3,63)=26.81, p<0.001]$ and on the time to perform the SRAT $[\mathrm{F}(3,63)=$ $4.1, p<0.01]$.

The post hoc tests revealed that for the Badcamp, the performance time was $4.48 \%$ shorter for badminton players $(14.07 \pm 0.84 \mathrm{~s})$ when compared to team sports players $(14.73 \pm$ $0.67 \mathrm{~s}), 7.55 \%$ shorter when compared to tennis players (15.22 $\pm 0.68 \mathrm{~s})$, and $15.08 \%$ shorter when compared to track and field athletes $(16.57 \pm 1.02$ s). There was no difference in the time to perform the Badcamp between team sport and tennis 
players, however, team sports players and tennis players performed the Badcamp $11.1 \%$ and $8.15 \%$ faster, respectively, than the track and field athletes.

Moreover, the post hoc tests did not reveal differences in time to perform the SRAT for badminton $(18.37 \pm 1.23 \mathrm{~s})$, team sport $(18.75 \pm$ 0.73s), and tennis players (18.19 $\pm 1.09 \mathrm{~s})$. However, the post hoc tests showed that the times to perform the SRAT were $5.21 \%$ and $6.14 \%$ shorter for badminton and tennis players, respectively, when compared to the track and field athletes (19.38 $\pm 1.04 \mathrm{~s})$.

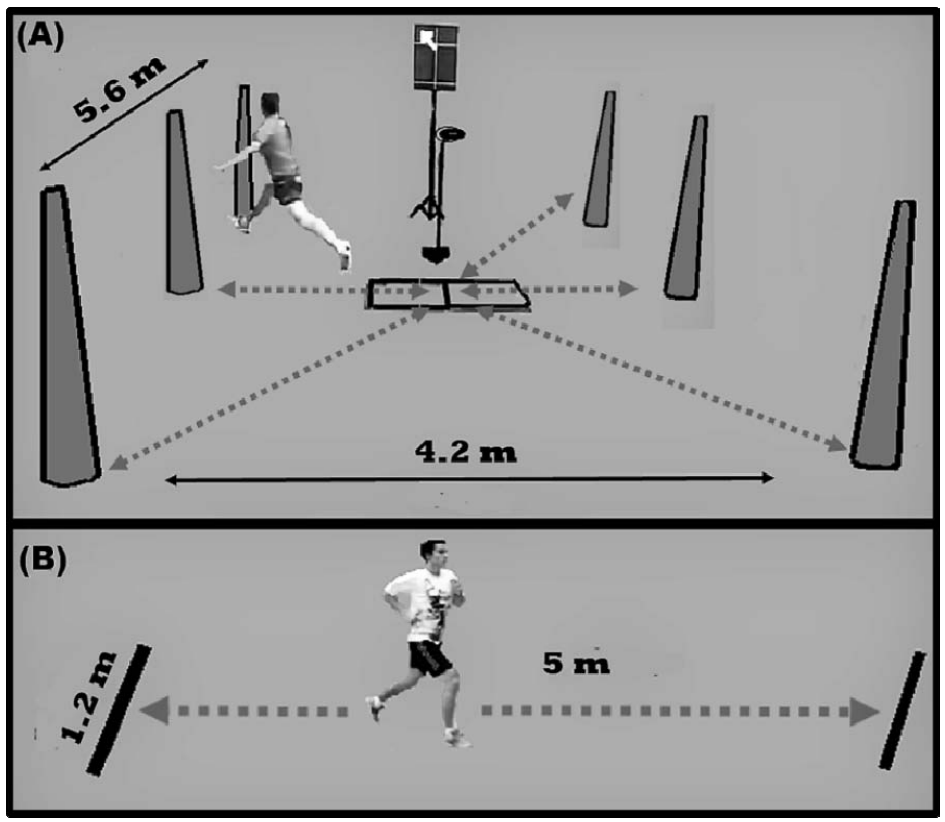

Figure 1

The Badcamp (A) and the shuttle run agility test (B) set up. Adapted from Loureiro Jr. and de Freitas, 2016.
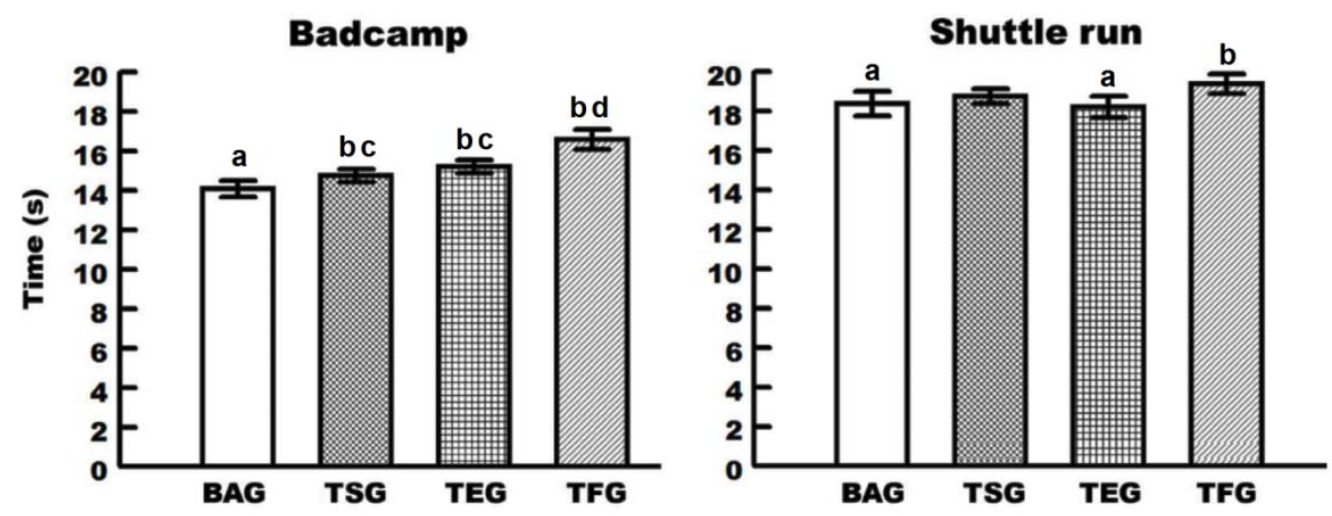

Figure 2

Performance times of badminton group (BAG), team sports group (TSG), tennis group (TEG,) and track and field (TFG) for the Badcamp test (left panel) and shuttle run (right panel). Statistical significance: $a<b$ and $c<d$. 


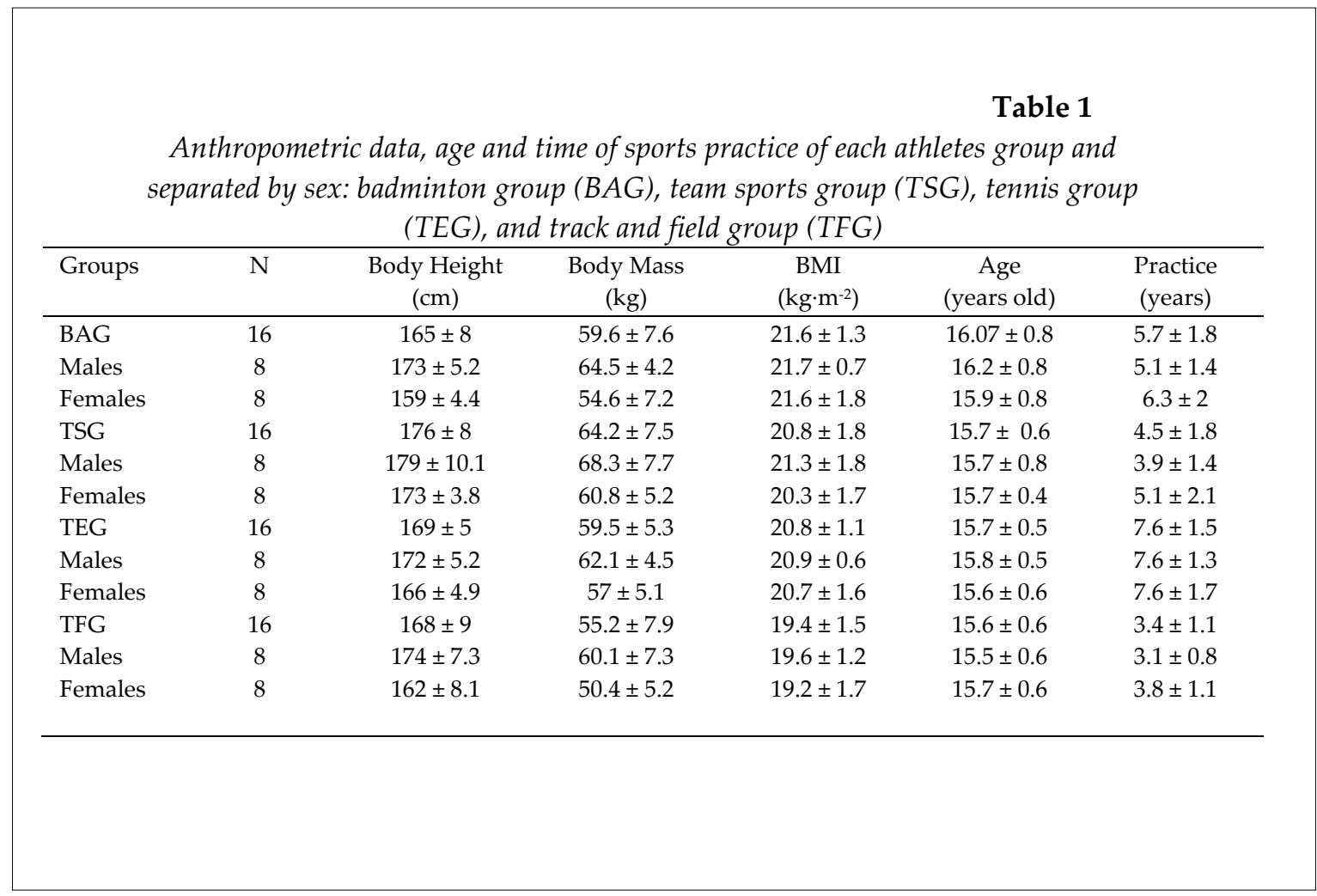

\section{Discussion}

We aimed with this study to examine the specificity of the Badcamp test for the agility assessment of badminton players. We hypothesized that badminton players would have better performance than athletes from other sports in the Badcamp, yet this performance would not be observed in the SRAT. The results supported the hypothesis, as while our results revealed that the group of badminton players showed better performance in the Badcamp than all other tested groups, in the SRAT the group of badminton players presented better performance only when they were compared to track and field athletes. Another interesting result is that the group of track and field athletes had the worst performance in both the Badcamp and SRAT tests.

From all the sport modalities selected for the study, the only one that is not considered an open skills sport is track and field. Therefore, it would be natural for track and field athletes to show inferior performance in tasks or tests requiring multiple stimulus and motor response possibilities. Corroborating our findings, another study revealed that tennis players had superior performance than swimmers when the task involved decision making activities (Wang et al., 2013). In addition, track and field events are performed by linear running and jumping. Thus, they do not include sudden body decelerations or changes of direction, unlike team and racquet sports. Studies have shown that linear sprints as well as lower limb muscle strength and power have no strong relationship with the tests involving changes of direction and deceleration (i.e. agility tasks) (Jones et al., 2009; Salaj and Markovic, 2011). Furthermore, a positive transfer of linear sprint training to performance in sprints with changes of direction has not been observed (Young et al., 2001), what would also explain the lower performance of the track and field athletes in the SRAT.

The results revealed that badminton players had the best performance in the Badcamp. They would have advantage in the decisionmaking process during the Badcamp due to the similarity of the test with the demands required in practice sessions and in competition. Regarding the perceptual matter, despite the visual stimuli are generic (i.e., lighting arrows), the way they are presented, simulating a badminton court and indicating the movement directions, makes this test similar to a routinely performed badminton 
drill known as "shadow badminton", in which the coach points out directions on the court (backcourt, midcourt, and frontcourt) so that the player performs a movement towards the indicated direction on the court. In accordance with our findings, another study revealed that elite baseball hitters had lower reaction times in a go/no go reaction time task than lower level baseball as well as tennis players, although no difference between groups was found when a simple reaction time task was performed (Kida et al., 2005). The explanation is that the go/no go task requires stimulus detection and interpretation in order to trigger the proper reaction (i.e., plan and execute or to plan and immediately inhibit the motor response) and the elite baseball hitters are more familiar and excel in solving this kind of problem. Indeed, elite baseball hitters are all the time challenged to decide, based on visual stimuli, whether the baseball is going towards or out the "strike zone", and consequently, execute or inhibit the planned motor response. Conversely, in the simple reaction task it does not happen as the stimulus is considered generic does not require signal interpretation and response selection and, consequently, does not challenge the decisionmaking processes of the tested athletes. In summary, we suggest that if the visual stimuli used in a test resemble practice or game situations, athletes from that specific sport would take advantage and have better performance than athletes from sports who are not used to receive and process such kind of stimuli. Similar rationale could be applied when expert and non-expert players from the same sport modality are compared against each other.

Another factor that could explain the best performance of badminton players in the Badcamp is the movement technique employed by them. Although there were no instructions regarding the movement athletes should make, badminton players adopted similar movements to the ones carried out in practice and during the matches and the same did not occur with other athletes. The movement techniques used in badminton have specific characteristics. Movements are initiated from the court central area and are composed of diagonal rapid steps towards the front and backcourt and side lunges (Hong et al., 2014). After years of training these specific movements, more efficient techniques resembling the ones performed in the Badcamp are learned. Also, those movements would require less time to be generated by the central nervous system as they have been performed many times throughout the players' life. Both aspects could give badminton players additional advantage in this test. Thus, the quicker the movement planning phase, the shorter the reaction time (Schmidt and Lee, 2011). Team sports (i.e. volleyball and basketball) and tennis also require rapid movements with changes of direction, yet the type of movement performed during practice and competition is mostly not related to movements performed in the Badcamp. In fact, in tennis most of the displacements consist of linear runs in the bottom of the tennis court, with few forward movements toward the net to volley and the same few backward movements. In volleyball, athletes mostly need to quickly react to fast balls during defensive plays, moving their body at short distances towards the ball or to stay in the best position to be an option to the setter in the attack. Basketball requires from players linear running during transition from defense to offense (and vice-versa), lateral sliding to control opponent's advance, and other movements not similar to the ones performed in the Badcamp.

In opposition, the lack of performance difference between groups of athletes who practice open skill sports (i.e., badminton, team sports and tennis players) during the SRAT could be explained by the fact that the movements performed during this test do not resemble movements performed in any of the tested sport modalities, as well as by the fact that there was no need to decide the running direction because in the SRAT the direction is previously determined. The tasks of running forward and turning by 180 degrees are performed in various types of activities carried out during the motor development and overall regular workouts used in various athletic training programs. Therefore, it makes actions performed in the SRAT common to all the tested groups. Likewise, the lack of difference in the SRAT between groups of open skill sports reduces the possibility that the results obtained in the Badcamp could be due to differences found between groups in anthropometric data, chronological age and time of sports practice. The only difference found was between badminton players and track and field 
athletes, and tennis players and track and field athletes. Specifically for badminton players, the difference between them and track and field athletes was only $5.2 \%$. In the Badcamp, this difference was $15.1 \%$. Those facts support the notion that the Badcamp is a specific test for badminton players.

This study has limitations that should be considered. The athletes of the team sport group were only basketball and volleyball players, which may not represent the global performance of athletes who play team sports such as football, handball, and rugby. In addition, we tested young athletes with age between 14 and 16 years. The results could have been different if professional and/or high level players had been evaluated. However, we believe that the difference in the Badcamp performance between high-level badminton players and athletes from other sports would be even greater than the difference found in this study.

\section{Conclusion}

Based on the findings of this study we may conclude that the Badcamp agility test, which was created to assess badminton players by simulating specific movements and conditions of uncertainty, is a specific agility test for badminton players given that the differences between badminton players and other groups of athletes occur only when these athletes have their agility evaluated by the Badcamp agility test. Thus, the Badcamp test should preferably be used by coaches and sport scientists to assess agility in badminton players in order to evaluate, for instance, the effectiveness of a particular training method or to detect talented individuals in this sport, considering that this specific test requires performance of this ability as it is really manifested on the court.

\section{Acknowledgements}

The authors would like to thank the Badminton World Federation (BWF) for the financial support and players and coaches for their time.

\section{References}

Adam C, Klissouras V, Ravazzolo M, Renson R, Tuxworth W. EUROFIT: European test of physical fitness. Rome: Council of Europe, Committee for the development of sport; 1988

Benvenuti C, Minganti C, Condello G, Capranica L, Tessitore A. Agility assessment in female futsal and soccer players. Medicina (Kaunas), 2010; 46: 415-420

Cheng Y, Whang Y, Liang C. Reaction time and competitive ability of badminton players. Zhejiang Sport Sci, 2006; 28: 60-63

Farrow D, Young $\mathrm{W}$, Bruce L. The development of a test of reactive agility for netball: a new methodology. J Sci Med Sport, 2005; 8: 52-60

Hong Y, Wang SJ, Lam WK, Cheung JTM. Kinetics of badminton lunges in four directions. J Appl Biomech, 2014; 30: 113-118

Hussain I, Arshad BM. Kinematical analysis of forehand and backhand smash in badminton. Innov Syst Des Eng, 2011; 2: 20-6

Jaitner T, Gawin W. A mobile measure device for the analysis of highly dynamic movement techniques. Procedia Eng, 2010; 2: 3005-3010

Jones P, Bampouras T, Marrin K. An investigation into the physical determinants of change of direction speed. J Sports Med Phys Fitness, 2009; 49: 97-104

Kida N, Oda S, Matsumura M. Intensive baseball practice improves the Go/No go reaction time, but not the simple reaction time. Cogn Brain Res, 2005; 22: 257-264

Little T, Williams AG. Specificity of acceleration, maximum speed, and agility in professional soccer players. J Strength Cond Res, 2005; 19: 76-78 
Loureiro Jr LFB, De Freitas PB. Influence of the performance level in badminton players in neuromotor aspects during a target-pointing task. Braz J Sports Med, 2012; 18: 203-207

Loureiro Jr LFB, De Freitas P.B. Development of an agility test for badminton players and assessment of its validity and test-retest reliability. Int J Sports Physiol Perform, 2016 11: 305-310

Mikolajec K, Maszczyk A, Zajac T. Game Indicators Determining Sports Performance in the NBA. J Hum Kinet, 2013; 37: 145-151

Monte A, Monte FG. Tests of agility, reaction speed and velocity for tennis. Rev Bras Cineantropom Desempenho Hum, 2007; 9: 401-407

Salaj S, Markovic G. Specificity of jumping, sprinting, and quick change-of-direction motor abilities. J Strength Cond Res, 2011; 25:1249-1255

Schmidt RA, Lee TD. Motor control and learning: a behavioral emphasis. $5^{\text {th }}$ Ed. Champaign: Human Kinetics; 2011

Sheppard JM, Young WB, Doyle TLA, Sheppard TA, Newton RU. An evaluation of a new test of reactive agility and its relationship to sprint speed and change of direction speed. J Sci Med Sport, 2006; 9: 342349

Sporis G, Jukic I, Milanovic L, Vucetic V. Reliability and factorial validity of agility tests for soccer players. J Strength Cond Res, 2010; 24: 679-686

Tiwari LM, Rai V, Srinet S. Relationship of selected motor fitness components with the performance of badminton player. Asian J Phys Educ Comput Sci Sports, 2011; 5: 88-91

Twist PW, Benicky D. Conditioning lateral movement for multi-sport athletes: Practical strength and quickness drills. Strength Cond J, 2010; 18: 10-19

Veale JP, Pearce AJ, Carlson JS. Reliability and validity of a reactive agility test for Australian football. Int J Sports Physiol Perform, 2010; 5: 239-248

Vescovi JD, Mcguigan MR. Relationships between sprinting, agility, and jump ability in female athletes. J Sports Sci, 2008; 26: 97-107

Wang $\mathrm{CH}$, Chang CC, Liang YM, Shih CM, Chiu WS, Tseng T, Hung DL, Tzeng OJL, Mugleton NG, Juan CH. Open vs. closed skill sports and the modulation of inhibitory control. PloS one, 2013; 8:e55773

Young WB, Mcdowell MH, Scarlett BJ. Specificity of sprint and agility training methods. J Strength Cond Res, 2001; 15: 315-319

\section{Corresponding author:}

\section{Paulo Barbosa de Freitas}

Instituto de Ciências da Atividade Física e Esportes - ICAFE

Universidade Cruzeiro do Sul

Rua Galvão Bueno, 868 São Paulo, SP, Brazil 01506-000

Phone number: +55 11 3385-3103

Fax number: +55 $113385-3103$

E-mail : paulo.defreitas@pq.cnpq.br and defreitaspb@gmail.com 